\title{
Understanding the formation of maxillary sinus in Japanese human foetuses using cone beam CT
}

\author{
Rieko Asaumi · Iwao Sato • Yoko Miwa • \\ Kosuke Imura • Masataka Sunohara • \\ Taisuke Kawai · Takashi Yosue
}

Received: 28 August 2009/Accepted: 6 May 2010/Published online: 21 May 2010

(C) The Author(s) 2010. This article is published with open access at Springerlink.com

\begin{abstract}
The formation of the maxillary sinus (MS) is tied to the maturation of the craniofacial bones during development. The MS and surrounding bone matrices in Japanese foetal specimens were inspected using cone beam computed tomography relative to the nasal cavity (NC) and the surrounding bones, including the palatine bone, maxillary process, inferior nasal concha and lacrimal bone. The human foetuses analysed were $223.2 \pm 25.9 \mathrm{~mm}$ in crown-rump length (CRL) and ranged in estimated age from 20 to 30 weeks of gestation. The amount of bone in the maxilla surrounding the MS increased gradually between 20 and 30 weeks of gestation. Various calcified structures that formed the bone matrix were found in the cortical bone of the maxilla, and these calcified structures specifically surrounded the deciduous tooth germs. By 30 weeks of gestation, the uncinate process of the ethmoid bone formed a border with the maxilla. The distance from the midline to the maximum lateral surface border of the MS combined with the width from the midline to the maximum lateral surface border of the inferior nasal concha showed a high positive correlation with CRL in Japanese foetuses. There appears to be a complex correlation between the MS and $\mathrm{NC}$ formation during
\end{abstract}

R. Asaumi - T. Kawai · T. Yosue

Department of Oral and Maxillofacial Radiology,

School of Life Dentistry at Tokyo, Nippon Dental University,

1-9-20 Fujimi Chiyoda-ku, Tokyo 102-8159, Japan

I. Sato $(\bowtie) \cdot$ Y. Miwa · K. Imura · M. Sunohara Department of Anatomy, School of Life Dentistry at Tokyo, Nippon Dental University, 1-9-20 Fujimi Chiyoda-ku,

Tokyo 102-8159, Japan

e-mail: iwaoa1@tokyo.ndu.ac.jp development in the Japanese foetus. Examination of the surrounding bone indicated that MS formation influences maturation of the maxilla and the uncinate process of the ethmoid bone during craniofacial bone development.

Keywords Cone beam CT - Maxillary sinus . Inferior nasal concha $\cdot$ Deciduous tooth $\cdot$ Development

\section{Introduction}

The maxillary sinus (MS) begins to form at 10 weeks of gestation during the process of primary pneumatisation, with the MS originating in the middle meatus and extending into the ethmoid cartilage. At 20 weeks of gestation, when secondary pneumatisation occurs, the MS then extends into the ossifying maxilla [16]. During foetal development, the MS enlarges to form the nasal cavity (NC) with the maxilla and other surrounding nasal bones. The morphology of the medial wall within the vertical plate of the palatine bone, the maxillary process, the inferior nasal concha and the lacrimal bone of the NC have been examined [16]. Tooth germs appear in the inferior borders of the maxillary prominences [7]. The palatine bone, inferior nasal concha, lacrimal bone and palatine process of the maxilla all play important roles in formation of the MS during development of the lower face. While previous studies have focused on the role of the paranasal sinuses and the morphology of the air sinuses in formation of the NC $[8,15,18]$, the involvement of matrix calcification in the surrounding bones in this process remains unknown. Hypoplasia of MS-associated malformation is related to ethmoidal air cells during formation of the NC [14]. Several types of hypoplasia of the MS are known, including hypoplasia and aplasia of 
the MS, MS septa, ethmomaxillary sinus, and superior meatus-draining MS and over-pneumatisation with respect to the MS septa and infraorbital fissure [13]. The effects of MS hypoplasia on other sinuses and the nasal cavity bones are currently unknown. The development of the teeth requires proportionate growth of the maxilla at the same time as when the growing maxilla is undergoing remodelling [3, 9]. The development of the paranasal sinuses is also connected to the growth of the facial cranium and teeth [5]. The morphology and formation of the MS are thought to be affected by the development of the maxilla and related bones, but little is known about the role of calcification and the resulting structure of the bone during development of the human foetus. Therefore, we studied the morphology of the MS and its accompanying structures using cone beam computed tomography (CBCT) to better understand the processes involved in facial development.

\section{Subjects and methods}

\section{Subjects}

This study was performed in 25 Japanese foetuses, which averaged $223.2 \pm 25.9 \mathrm{~mm}$ in crown-rump length (CRL) and ranged in estimated age from 20 to 30 weeks of gestation. The approximate age of each foetus was determined using Streeter's tables [17]: 20 weeks, 175-186 mm; 24 weeks, 217-228 mm; 30 weeks, 275-284 mm. The CRL was determined by straightening the foetal body and placing the head so that the eye-ear line was perpendicular to the axis of the body.

\section{Methods}

\section{CBCT images}

Cone beam computed tomography (PSR 9000N, Asahi Roentgen Industry, Kyoto, Japan) was used in this study. Images of the MS and surrounding structures were acquired for 25 foetuses (15 males and 10 females). The CBCT was operated at a tube potential of $60 \mathrm{kV}$ and tube current of $4 \mathrm{~mA}$, and the scans acquired cylindrical areas of $\phi$ $41 \times 40 \mathrm{~mm}$ with high resolution (voxel size $=0.1 \mathrm{~mm}$ ). From the three-dimensional CBCT images, the diameter of the MS was measured using ASAHI vision software (Asahi Roentgen Industry) and Micro AVS version 11 software (KGT, Tokyo, Japan). After identifying the median sagittal plane and the palatal plane (PP), which is defined by the anterior and posterior nasal spines and is perpendicular to the median sagittal plane, as a reference plane, the measurements described below were performed.

\section{Measurements}

The points measured in the CBCT images of the Japanese foetuses are shown in Fig. 1. Four measurements of the maxilla with respect to the tooth germ were made: $\mathrm{Dh}$ is the height of the first deciduous molar in the maxilla from the PP to the superior border of the dental sac (Fig. 1a); Dw is the width of the first deciduous molar in the maxilla from the midline to the inner border of the dental sac (Fig. 1a); $\mathrm{Eh}$ is the height of the second deciduous molar in the maxilla from the PP to the superior border of the dental sac (Fig. 1b); Ew is the width of the second deciduous molar in the maxilla from the midline to the inner border of the dental sac (Fig. 1b).

In addition, three measurements of the maxillary sinus were made: INMw is the width from the midline to the lateral (outer) surface border of the inferior nasal concha (Fig. 1c); MSw is the width from the midline to the lateral (outer) surface border of the MS (Fig. 1D); MSh is the height from the PP to the inferior border of the MS (Fig. 1d).

\section{Ethics}

The foetuses were obtained from spontaneous abortions with the consent of each family in accordance with the Law Concerning Body Donation for Medical and Dental Education and the Law Concerning Cadaver Dissection and Preservation. The foetuses were all examined at Nippon Dental University.

\section{Statistical methods}

Linear regression analyses to correlate the CRL and facial growth were performed. Pearson's correlation coefficient was used to determine the correlations.

\section{Results}

The linear regression equations for maxillary sinus growth (MSG) are shown in Fig. 2a-g. There was a highly positive correlation between the MSG (MSw, $r=0.861$ : INMw, $r=0.820$ ) and the CRL (Fig. 2f, g). The correlations between the CRL and Ew $(r=0.600)$, MSh $(r=0.577)$ and Eh $(r=0.682)$ were moderately positive (Fig. 2b, d, e), while the correlations between the CRL and Dh $(r=$ $0.424)$ and Dw ( $r=0.423$ ) were low (Fig. 2a, c).

The growth pattern of the facial bones between 20 and 30 weeks of gestation was examined using three-dimensional reconstruction of a series of CBCT images (Figs. 3, $4,5)$. From the frontal view, the maxilla was located at the inner lower part of the orbit at 20 weeks of gestation. 
Fig. 1 Frontal view showing the measurement points of the maxillary sinus in the maxilla on CBCT images. PP, defined by the anterior and posterior nasal spines and is perpendicular to the median sagittal plane; $\mathrm{Dh}$, height of the first deciduous molar in the maxilla from the PP to the superior border of the dental sac; Dw, width of the first deciduous molar in the maxilla from the midline to the inner border of the dental sac; Eh, height of the second deciduous molar in the maxilla from the PP to the superior border of the dental sac; Ew, width of the second deciduous molar in the maxilla from the midline to the inner border of the dental sac; INMw, width from the midline to the lateral (outer) surface border of the inferior nasal concha; MSh, height to the inferior border of the maxillary sinus at a right angle from the $\mathrm{PP}$; MSw, width from the midline to the lateral (outer) surface border of the maxillary sinus
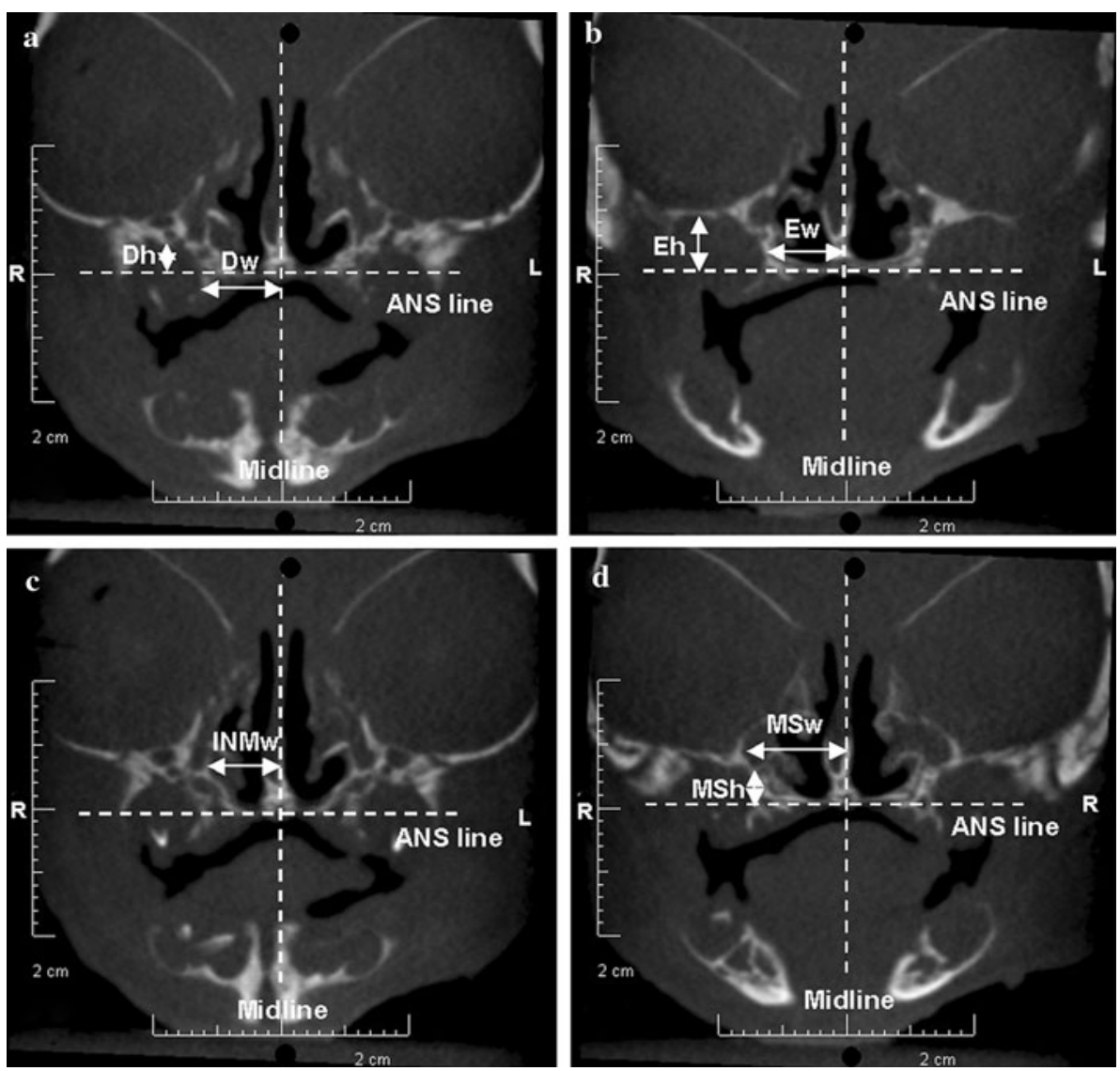

The small tooth germ of the deciduous cuspid in the maxilla was located on the outer lower part of the maxilla. The maxilla, zygomatic bone and vomer could also be visualised at this age (Fig. 3a1, a2, b1, b2). In contrast, the nasal basal region of a thin part of the $\mathrm{V}$-shaped vomer could not be visualised clearly at this gestational age, as these structures were faint in some CBCT images and not clear in other images (Figs. 4a1, a2, b1, b2; 5a1, a2, b1, b2). The amount of bone within the vomer increased gradually, proceeding from the surface of the palatal process of the maxilla toward the vertical plate (Figs. 3, 4).

At 20 weeks of gestation, the MS appeared as a small space basal to the lateral side of the middle concha in the NC (Fig. 3a1, a2, b1, b2).

At 30 weeks of gestation, a region of radiopacity in the lower part of the anterior border of the vomer could be seen in the CBCT images (Fig. 5a1, a2, b1, b2). When examined horizontally at a gestational age of 20 weeks, the vomer appeared in two separate bone matrix layers (Fig. 3a1, a2, b1, b2). In contrast, the vertical plate of the ethmoid bone still had a low density in comparison with the other bone matrices in the NC at 30 weeks of gestation (Fig. 5a1, a2, b1, b2).

The palatal process of the maxilla appeared in the CBCT images at 24 weeks of gestation. The thin lateral zone of the zygomatic bone widened gradually and appeared to have a higher density than the surface of the maxilla where the cleft formed on the lateral side of the radiopaque zone around the infraorbital canal (Fig. 4a1, a2, b1, b2). In the maxilla, the area around the infraorbital canal and the cleft of the maxillary sinus were observed clearly on the surface of the $\mathrm{NC}$ at this gestational age (Fig. 4a1, a2, b1, b2). The lateral surface of the maxilla was radiopaque at 30 weeks of gestation (Fig. 5a1, a2, b1, b2). The ethmoid bone and inferior nasal concha all had low densities at 20 weeks of gestation, but their radiopacity increased gradually by 24 weeks of gestation (Figs. 3, 4). The lateral border of the bone matrix around the dental follicle of the teeth appeared at 24 weeks of gestation, and the radiopacity in the CBCT images increased gradually as the bone matrix developed. The tooth germ of the first deciduous molar was located in the lateral lower part of the maxilla, and the deciduous incisors and canines, which showed partial calcification of the crowns, were also located under this border (Figs. 3, 4, 5).

\section{Discussion}

The CBCT images clearly showed calcification of the bone matrix and the side of the cortical bone during the 

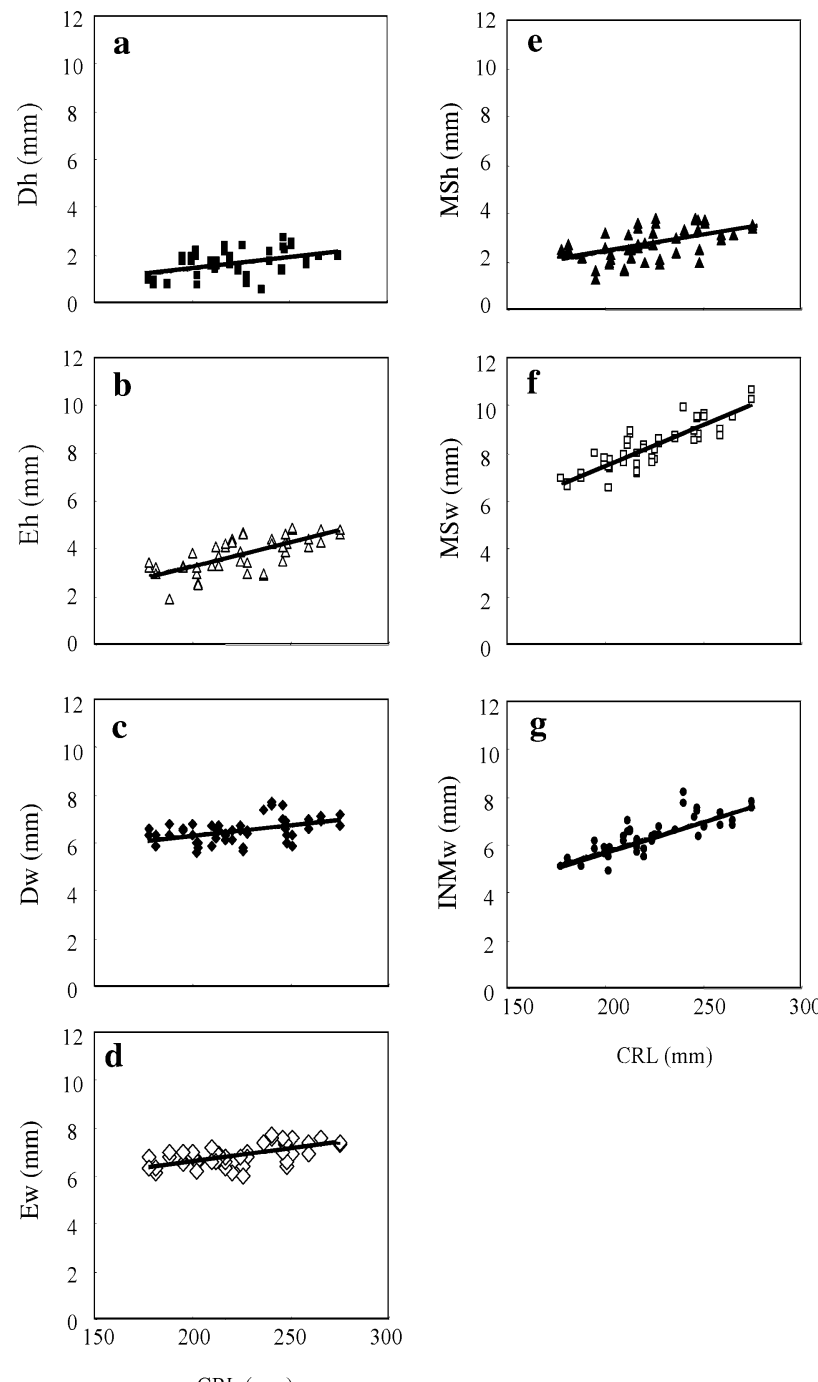

CRL (mm)

Fig. 2 Regression lines (black lines) for the correlation between CRL and growth of facial length (Fig. 1). Pearson's correlation coefficient was used to measure the strength of the association between the two variables. MSG (MSw, $r=0.861$ : INMw, $r=0.820$ ); $\mathrm{Ew}$ $(r=0.600)$; MSh $(r=0.577)$; Eh $(r=0.682)$; Dh $(r=0.424)$; Dw $(r=0.423)$

formation and development of the maxillary sinus (MS) in the human foetus throughout the period from gestational weeks 20 to 30. The growth rate of facial features relative to component weight was similar to that of the body weight in Brazilian foetuses reported previously [12]. The length and area associated with the cranial base were related to the CRL in Korean foetuses [10]. Our data showed an increased growth rate of the CRL relative to the growth of facial features in Japanese foetuses with respect to width, as indicated by several of our measurements, including MSw and INMw (Fig. 2a-g). Therefore, MS growth (MSG) is affected by the width of the NC and the lateral border of the maxilla. Kopper et al. [8] reported that the MS expands not only in the posterior direction, but also
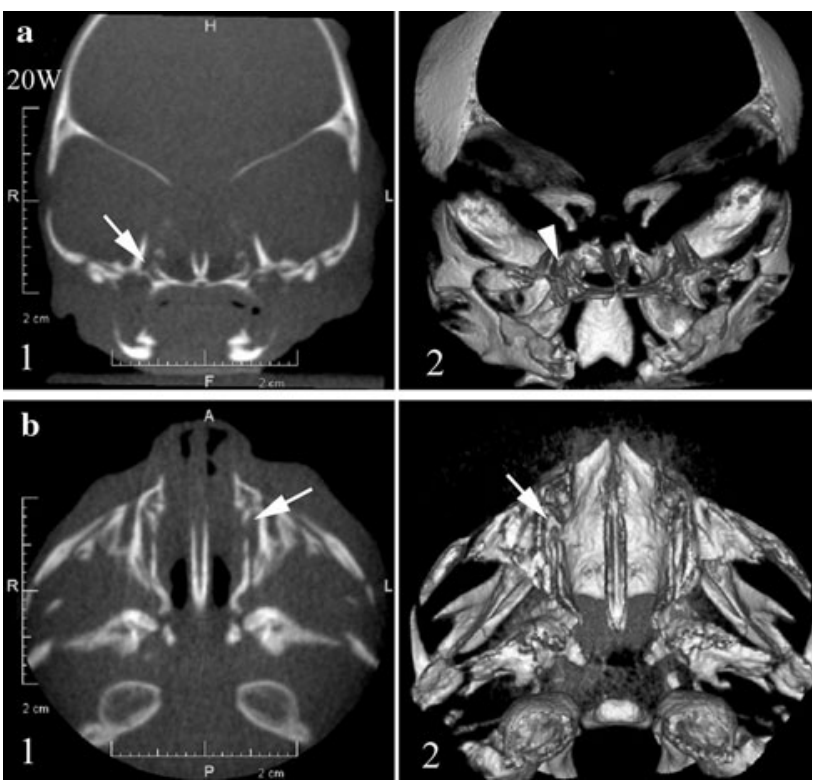

Fig. 3 Three-dimensional reconstruction of the nasal wall in the lower facial region at 20 weeks of gestation (CRL, $178 \mathrm{~mm}$ ). a Frontal view of the maxillary sinus and nasal cavity. The locations of the cross-sectional axial slices that were taken through the area of interest are indicated (a1). Three-dimensional frontal view of the maxillary sinus and nasal cavity. A density indicating the presence of the uncinate process of the ethmoid cartilage appeared near the nasal side of the maxilla (a2). b Horizontal view of the maxillary sinus and nasal cavity. The locations of the cross-sectional axial slices that were taken through the area of interest are indicated (b1). Threedimensional horizontal view of the maxillary sinus and nasal cavity (b2). Bar $2 \mathrm{~cm}$, arrow maxillary sinus, arrowhead uncinate process

outward in the anterior direction after it first appears and that the MS volume at a gestational age of 9 weeks is very small in Japanese foetuses. MS hypoplasia, which occurs at a frequency of approximately $4.2 \%$ [14] to $4.6 \%$ [13], is responsible for some abnormalities in the formation of the MS. The uncinate process of the ethmoid bone, which appears during the tenth week of gestation, is an important structure along with the MS septa for the proper formation of the paranasal sinuses $[1,4,13]$. The matrices within the maxilla begin the process of mineralisation at 20 weeks of gestation [16]. The sphenoid sinuses have a more variable effect, especially when compared to that of the MS, on development of the paranasal sinuses [15]. During weeks 11 and 12, the sinusoidal air space develops progressively lateral to the cartilaginous uncinate process from this space. The appearance of the uncinate process seems to influence the development of the MS at an early stage [2]. When invagination of mucosa invades the maxilla at 17 weeks of gestation, the appearance of the MS can then be correlated with formation of the lateral nasal wall [1]. The densities of the vomer, ethmoid bone, zygomatic bone, inferior nasal concha and maxilla were variable over the course of this study, indicating different levels of 

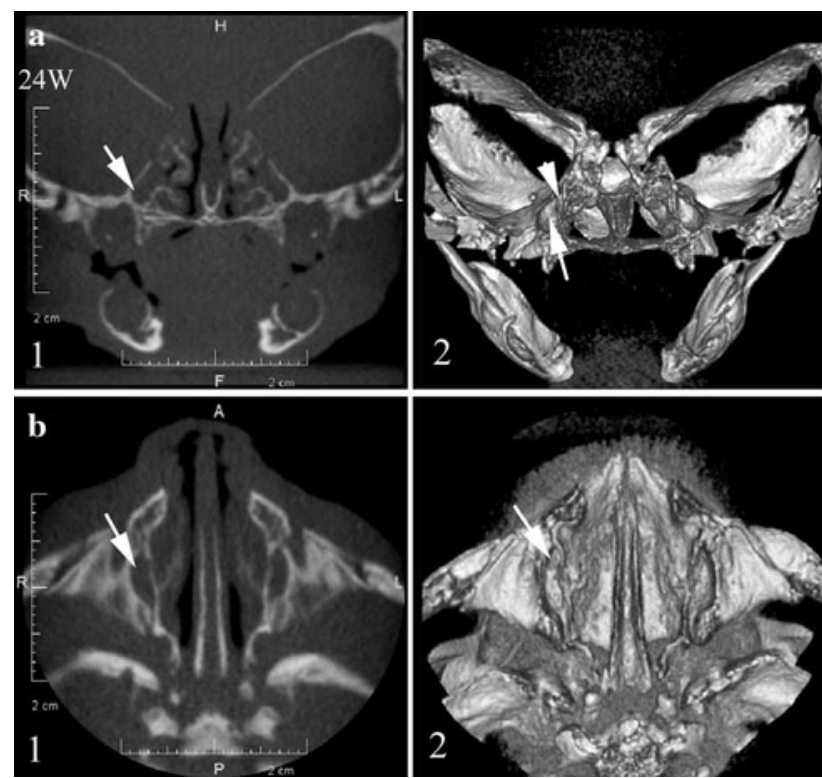

Fig. 4 Three-dimensional reconstruction of the nasal wall in the lower facial region at 24 weeks of gestation (CRL, $210 \mathrm{~mm}$ ). a Frontal view of the maxillary sinus and nasal cavity. The locations of the cross-sectional axial slices that were taken through the area of interest are indicated (a1). Three-dimensional frontal view of the maxillary sinus and nasal cavity. The uncinate process of the ethmoid bone is located near the nasal side of the maxilla (a2). b Horizontal view of the maxillary sinus and nasal cavity. The locations of the cross-sectional axial slices taken through the area of interest are indicated (b1). Three-dimensional horizontal view of the maxillary sinus and nasal cavity (b2). Bar $2 \mathrm{~cm}$, arrow maxillary sinus, arrowhead uncinate process

calcification in each of these structures at each stage during development. Parts of the maxilla, such as the lateral, palatine process of the maxilla, were clearly visible in the images at 24 weeks of gestation. This indicates that the MS is surrounded by bony structures comprised of some of the nasal bones at this stage. The uncinate process of the ethmoid bone was also seen clearly with a measurable density at 20 weeks of gestation. By 30 weeks of gestation, this process forms a border with the maxilla on the nasal side. The elongated structure of the uncinate process affects formation of the boundary on the nasal side between the maxilla and MS during development (Fig. 6). The MS also descends following the direction of the palatal process of the maxilla. This correlation is also related to the growth of facial width. Therefore, the formation and development of the MS are affected by nasal cavity elements, such as the uncinate process, vomer and maxilla, whereas the development of the $\mathrm{NC}$ is affected by different bones in the human foetus. In general, the bones of the facial skeleton developed at a slower rate than those of the calvariae due to the increase in volume of the foetal brain during the second and third trimesters [11]. However, the facial growth may change in the horizontal direction from 19 to 30 weeks
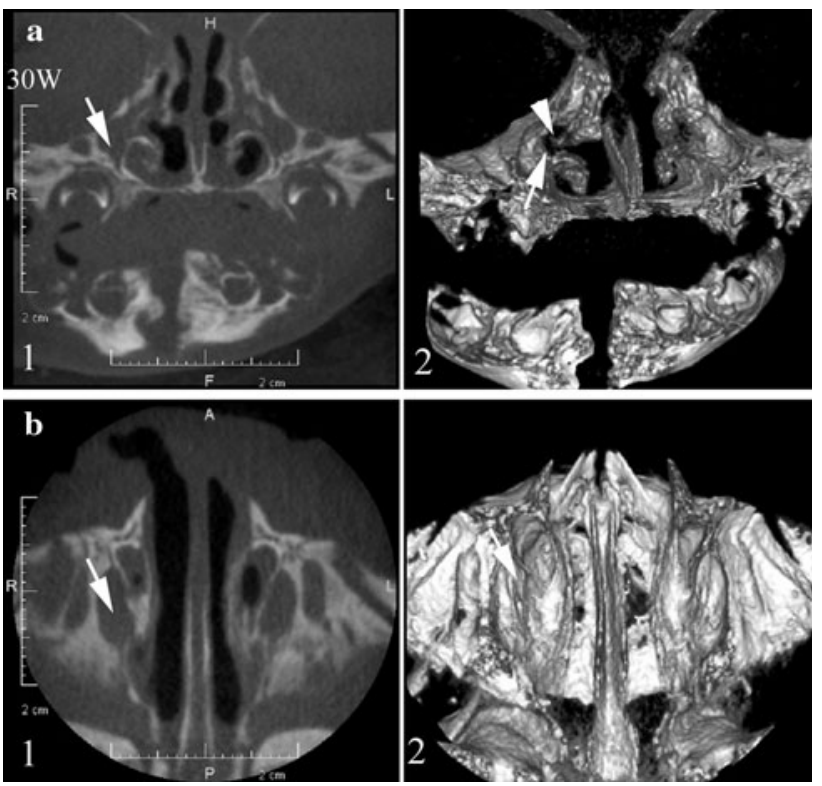

Fig. 5 Three-dimensional reconstruction of the nasal wall in the lower facial region at 30 weeks of gestation (CRL, $275 \mathrm{~mm}$ ). a Frontal view of the maxillary sinus and nasal cavity. The locations of the cross-sectional axial slices that were taken through the area of interest are indicated (a1). Three-dimensional frontal view of the maxillary sinus and nasal cavity. The uncinate process of the ethmoid bone formed a border with the maxilla (a2). b Horizontal view of the maxillary sinus and nasal cavity. The locations of the cross-sectional axial slices that were taken through the area of interest are indicated (b1). Three-dimensional horizontal view of the maxillary sinus and nasal cavity (b2). Bar $2 \mathrm{~cm}$, arrow maxillary sinus, arrowhead uncinate process

gestation. The highest growth rate was in the width of the NC [6]. In Korean foetuses, the maxilla grew in greater increments in the horizontal direction when compared to the vertical direction [10, 19]. Brazilian foetuses had a slower growth rate of the lower face in contrast to those of the zygomatic, maxilla and ethmoid bones [12]. Our cohort of Japanese foetuses had a more pronounced growth rate for the lower cranial bones and $\mathrm{NC}$ in the horizontal direction, which is similar to the observations in Korean foetuses $[10,19]$. Our data also indicated that the CRL had an increased growth rate when compared to the maxillary bone in the horizontal direction. These features that influence development of the craniofacial bones may characterise individuals of Asian descent. In addition, the bony space within the maxilla decreased gradually in response to elongation of the maxillary sinus during development of the dental sac. The roots of the teeth are located close to the lower floor of the MS and can be found outside the maxilla by 30 weeks of gestation. The roots of the first and second molars are located in close proximity to the lower floor of the MS. During formation of the maxilla, the tooth germs require space within the maxilla in which to elongate or expand as they also mature [7]. The development of the 
Fig. 6 Images of the maxilla and other craniofacial bones around the maxillary sinus during development from 20 to 30 weeks of gestation (CRL, $223.2 \pm 25.9 \mathrm{~mm}$ ). The uncinate process of the ethmoid bone extends to the maxillary sinus during development. Bar $1 \mathrm{~cm}$, a 20 weeks, b 24 weeks, c 30 weeks of gestation, $D C$ dental crypt, $E B$ ethmoid bone, $I N C$ inferior nasal concha, $M S$ maxillary sinus, $N S$ nasal septum
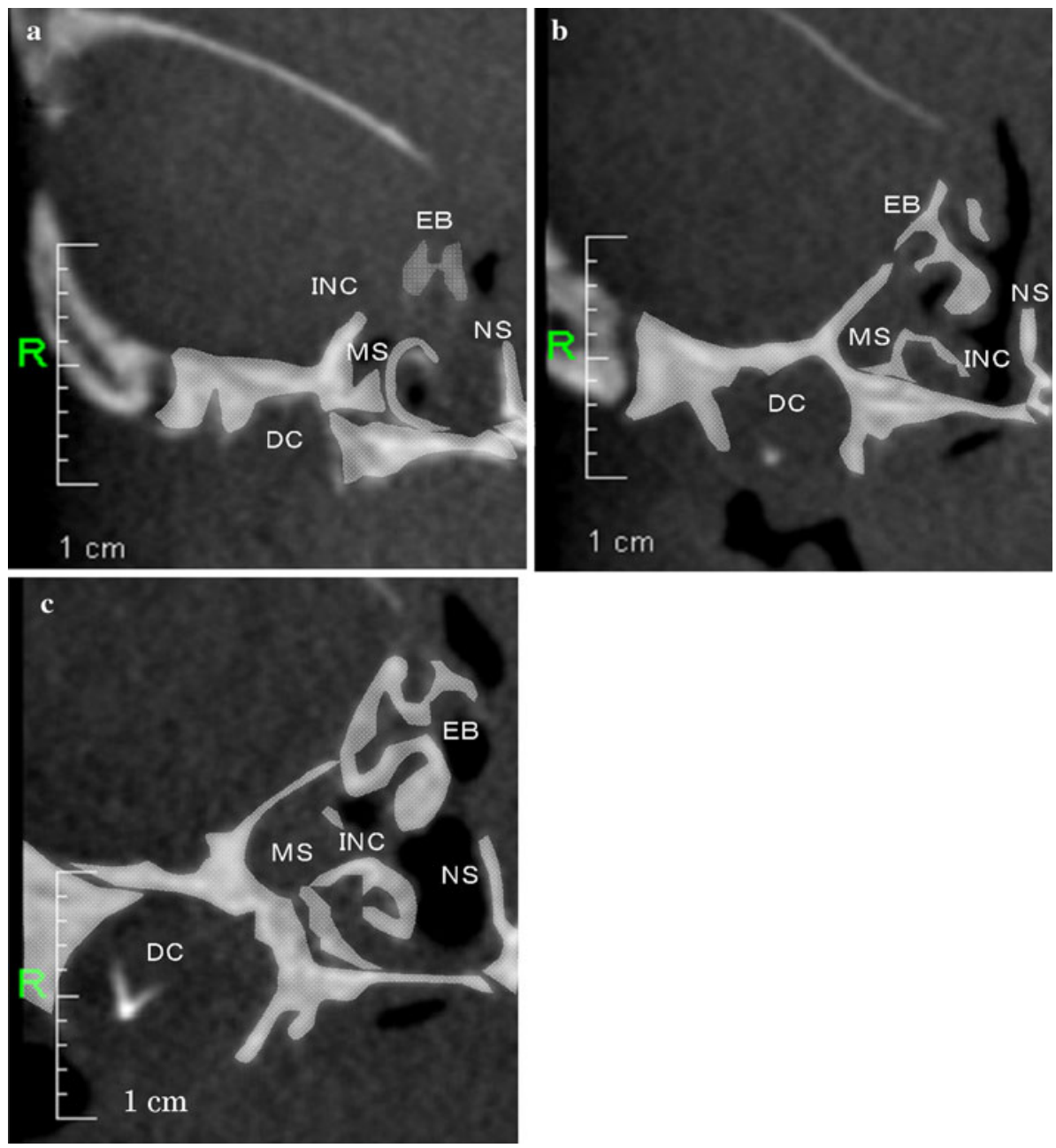

tooth germ, with their accompanying dental sacs, also affects the formation and development of the maxilla, especially with respect to the dimension of facial growth. As a result, the lower facial region containing the maxilla was altered by an elongated maxillary sinus and by the developing tooth germs in Japanese foetuses.

Open Access This article is distributed under the terms of the Creative Commons Attribution Noncommercial License which permits any noncommercial use, distribution, and reproduction in any medium, provided the original author(s) and source are credited.

\section{References}

1. Arredondo de Arreola G, López Serna N, de Hoyos Parra R, Arreola Salinas MA (1996) Morphogenesis of the lateral nasal wall from 6 to 36 weeks. Otolaryngol Head Neck Surg 114:54-60

2. Bingham B, Wang RG, Hawke M, Kwok P (1991) The embryonic development of the lateral nasal wall from 8 to 24 weeks. Laryngoscope 101:992-997

3. Enlow DH, Bang S (1965) Growth and remodeling of the human maxilla. Am J Orthod 51:446-464
4. Erdem T, Aktas D, Erdem G, Miman MC, Ozturan O (2002) Maxillary sinus hypoplasia. Rhinology 40:150-153

5. Hengerer AS (1984) Embryologic development of the sinuses. Ear Nose Throat J 63:134-136

6. Kanagawa T, Fukuda H, Kinugasa Y, Son M, Shimoya K, Murata Y, Yokoyama T, Hashimoto H, Isaka S, Nagamatsu M (2006) Mid-second trimester measurement of fetal nasal bone length in the Japanese population. J Obstet Gynaecol Res 32:403-407

7. Kitamura H (1989) Chapter 1. Early development of teeth. In: Kitamura $\mathrm{H}$ (eds) Embryology of the mouth and related structure. Maruzen Co. Ltd, Tokyo, pp 1-34

8. Koppe T, Yamamoto T, Tanaka O, Nagai H (1994) Investigations on the growth pattern of the maxillary sinus in Japanese human fetuses. Okajimas Folia Anat Jpn 71:311-318

9. Kurihara S, Enlow DH, Rangel RD (1980) Remodeling reversals in anterior parts of the human mandible and maxilla. Angle Orthod 50:98-106

10. Lee SK, Kim YS, Jo YA, Seo JW, Chi JG (1996) Prenatal development of cranial base in normal Korean fetuses. Anat Rec 246:524-534

11. Mandarim-de-Lacerda CA, Alves MU (1992) Growth of the cranial bones in human fetuses (2nd and 3rd trimesters). Surg Radiol Anat 14:125-129

12. Mandarim-de-Lacerda CA, Urania-Alves M (1993) Growth allometry of the human face: analysis of the osseous component 
of the mid and lower face in Brazilian fetuses. Ann Anat 175:475-479

13. Selcuk A, Ozcan KM, Akdogan O, Bilal N, Dere H (2008) Variations of maxillary sinus and accompanying anatomical and pathological structures. J Craniofac Surg 19:159-164

14. Sirikçi A, Bayazit Y, Gümüsburun E, Bayram M, Kanlikana M (2000) A new approach to the classification of maxillary sinus hypoplasia with relevant clinical implications. Surg Radiol Anat 22:243-247

15. Smith TD, Siegel MI, Mooney MP, Burrows AM, Todhunter JS (1997) Formation and enlargement of the paranasal sinuses in normal and cleft lip and palate human fetuses. Cleft Palate Craniofac J 34:483-489
16. Sperber GH (2001). 11 Pranasal sinues. In: Craniofacial development. BC Decker Inc, Hamilton/London, pp 123-125

17. Streeter GL (1951) Development horizons in fuman embryos description of age group XIX, XX, XXI, XXII and XXIII. Contrib Embryol 230:167-196

18. Vidic B (1971) The morphogenesis of the lateral nasal wall in the early prenatal life of man. Am J Anat 130:121-139

19. Yang WS, Suhr CH, Nahm DS, Chang YI (1990) Soft X-ray study on fetal cranio-facial growth and development. Taehan Chikkwa Uisa Hyophoe Chi 28:265-277 